\title{
A PROPERTY OF FREE BOOLEAN ALGEBRAS
}

ALFRED HORN ${ }^{1}$

Consider the following properties of a Boolean algebra $A$ :

$P_{1}$ : Every set of pairwise disjoint elements of $A$ is countable.

$P_{2}$ : Every chain in $A$ is countable.

For arbitrary Boolean algebras neither of these properties implies the other. The algebra of all sets of integers satisfies $P_{1}$ but not $P_{2}$, while the algebra of all finite or cofinite sets of real numbers satisfies $P_{2}$ but not $P_{1}$. It is well known that $P_{1}$ holds in any free Boolean algebra. However it is not generally realized that $P_{2}$ also holds in free Boolean algebras. In fact this statement has not explicitly appeared in the literature, although it is a consequence of a theorem in topology due to N. A. Sanin [1, Theorem 50]. The following is a simple direct proof of the statement.

TheOREM. Every chain in a free Boolean algebra is countable.

Proof. Let $T$ be a set of cardinality $\alpha$ and let $M$ be the set of all functions on $T$ with values 0 or 1 . For each $t \in T$ let $D_{t}$ $=\{f \in M: f(t)=1\}$. The algebra $F$ generated by the sets $D_{t}$ is the free Boolean algebra with $\alpha$ free generators. Now suppose $C$ is an uncountable chain in $F$. We may assume $0 \notin C$. For each member $x$ of $C$ there is a finite subset $A$ of $T$ such that $x$ is in the subalgebra generated by $\left\{D_{t}: t \in A\right\}$. Therefore there exist distinct subsets $A_{1}, \cdots, A_{r}$ of $A$ such that

$$
x=\bigcup_{i=1}^{r}\left[\bigcap_{t \in A_{i}} D_{t} \cap \bigcap_{t \in A-A_{i}} \bar{D}_{t}\right] .
$$

Thus to each member of $C$ we can associate a pair $(n, r)$ of positive in tegers, where $n$ is the cardinality of $A$, and $r$ is the number of subsets $A_{i}$. Since $C$ is uncountable there will certainly be distinct members $x$ and $y$ of $C$ associated with the same pair $(n, r)$. Then there exist subsets $A$ and $B$ of $T$ with cardinality $n$, distinct subsets $A_{1}, \cdots, A_{r}$ of $A$, and distinct subsets $B_{1}, \cdots, B_{r}$ of $B$ such that (1) holds, and

$$
y=\bigcup_{i=1}^{r}\left[\bigcap_{t \in B_{i}} D_{t} \cap \bigcap_{t \in B-B_{i}} \bar{D}_{t}\right] .
$$

Since $C$ is a chain we may assume $x \subset y$. We will reach a contradiction by showing $x=y$.

Received by the editors November 1, 1966.

1 This research was supported in part by National Science Foundation Grant No. 5600 . 
If $f$ is any member of $M$ and $S=\{t: f(t)=1\}$, then $f \in x$ if and only if $S \cap A=A_{i}$ for some $i$. Since $x \subset y$, it follows that for any subset $S$ of $T$,

(3) if $S \cap A=A_{i}$ for some $i$, then $S \cap B=B_{j} \quad$ for some $j$.

Let $C_{1}, \cdots, C_{p}$ be the distinct members of $\left\{A_{i} \cap B: 1 \leqq i \leqq r\right\}$ and $q$ be the cardinality of $A-B$, which is also equal to the cardinality of $B-A$. If $K$ is any subset of $B-A$, then by (3) with $S=A_{i} \cup K$, we see that for each $k, 1 \leqq k \leqq p$, there is a $j$ such that $K \cup C_{k}=B_{j}$. Therefore $r \geqq p \cdot 2^{q}$. However we also have $r \leqq p \cdot 2^{q}$, since each $A_{i}$ is of the form $C_{k} \cup K$ for some $k$, and some subset $K$ of $A-B$. Thus $r=p \cdot 2^{q}$, and hence

(4) $\left\{B_{i}: 1 \leqq i \leqq r\right\}=\left\{C_{k} \cup K: 1 \leqq k \leqq p\right.$ and $\left.K \subseteq B-A\right\}$

and

(5) $\left\{A_{i}: 1 \leqq i \leqq r\right\}=\left\{C_{k} \cup K: 1 \leqq k \leqq p\right.$ and $\left.K \subseteq A-B\right\}$.

Let $D=A \cap B$. Then by (2) and (4),

$$
\begin{aligned}
y & =\bigcup_{k=1}^{p} \bigcup_{K \subseteq B-A}\left[\bigcap_{t \in C_{k} \cup K} D_{t} \cap \bigcap_{t \in B-\left(C_{k} \cup K\right)} \bar{D}_{t}\right] \\
& =\bigcup_{k=1}^{p}\left[\bigcap_{t \in C_{k}} D_{t} \cap \bigcap_{t \in D-C_{k}} \bar{D}_{t}\right] \cap \bigcup_{K \subseteq B-A}^{\cup}\left[\bigcap_{t \in K} D_{t} \cap \bigcap_{t \in(B-A)-K} \bar{D}_{t}\right] \\
& =\bigcup_{k=1}^{p}\left[\bigcap_{t \in C_{k}} D_{t} \cap \bigcap_{t \in D-C_{k}} \bar{D}_{t}\right] .
\end{aligned}
$$

Similarly

$$
x=\bigcup_{k=1}^{p}\left[\bigcap_{t \in C_{k}} D_{t} \cap \bigcap_{t \in D-C_{k}} \bar{D}_{t}\right]
$$

and so $x=y$.

\section{REFERENCE}

1. N. A. Šanin, O proizvedenii topologičlskih prostranstv, Trudy Mat. Inst. Steklov. 24 (1948), 112 pp.

University of California, Los Angeles 